\title{
Bilateral Facial Palsy and Tuberculous Otitis Media: Case Report
}

\author{
Costa RR*, Lourençone LFM, Marchiori R, J orge \\ J C, Oliveira EB and Brito R \\ Department of Otorhinolaryngology, Hospital de \\ Reabilitação de Anomalias Craniofaciais, USP, Brazil \\ *Corresponding author: Costa RR, Department \\ of Otorhinolaryngology Hospital de Reabilitação de \\ Anomalias Craniofaciais, USP, Brazil
}

Received: April 06, 2018; Accepted: May 04, 2018; Published: May 11, 2018

\begin{abstract}
The purpose of the study is to report a rare case of bilateral tuberculous otitis media complicated by suppurative labyrinthitis and bilateral peripheral facial paralysis in a 74-year-old female patient. The patient had intense otalgia associated with bilateral dense and purulent otorrhea, incapacitating rotational vertigo and peripheral facial paralysis, initially left with progression to the right, House Brackman IV and V respectively, with about 60 days of evolution, being submitted in other services to antibiotic therapy with ceftriaxone, sulfamethoxazole-trimethoprim and ciprofloxacin, without clinical resolution.
\end{abstract}

From additional clinical findings such as chronic cough, chronic suppurative joint injury and recurrent urinary tract infection, we undertook investigation with computed tomography, nuclear magnetic resonance, biochemistry, serology and BK sputum test, and this last one with positive result on all three samples.

Confirming the diagnosis of tuberculosis, which associated with others clinical findings, was defined as tuberculous otitis media, the treatment of the Brazilian protocol was begun. This consisting of rifampicin, isoniazid, pyrazinamide and ethambutol for 2 months, and rifampicin and isoniazid for a further 4 months. Corticoid therapy was used for 10 days.

There were significant improvements of facial paralysis throughout the first 15 days of treatment, in addition to progressive vestibular compensation with return to daily life activities.

Keywords: Tuberculous Otitis Media; Bilateral Facial Palsy; Suppurative Labyrinthitis; Facial Palsy

\section{Case Presentation}

A 74-year-old female patient with no prior comorbidities had severe otalgia associated with bilateral and severe purulent otorrhea, severe right and left mixed hearing loss, disabling rotatory vertigo and peripheral facial palsy, initially left with right progression, House Brackmann (HB) classification V to the right and IV to the left, with 60 days of evolution. She had being submitted in other services to antibiotic therapy with ceftriaxone, sulfamethoxazole-trimethoprim and ciprofloxacin, without resolution of the condition.

Based on additional clinical findings such as chronic cough, chronic suppurative joint injury and recurrent urinary tract infection, a research was performed with tomography of temporal bones and thorax, skull resonance, serum and urinary biochemistry, serology, and BK sputum and urine screening, with a positive test for the presence of alcohol-acid resistant bacillus through PCR method in the three sputum samples and in the urine sample, confirming the diagnosis of pulmonary and renal tuberculosis, as well as right shoulder imaging evidencing signs of destructive arthritis associated with calcifications articular and periarticular lesions compatible with tuberculosis. All the findings associated with the clinical condition were defined as tuberculous otitis media.

The Brazilian treatment protocol for tuberculosis was initiated, which consists of rifampicin, isoniazid, pyrazinamide and ethambutol for 2 months and rifampicin and isoniazid for another 4 months, associated with corticosteroid therapy for 10 days.

The patient progressed with suppuration regression and significant improvement of facial palsy throughout the first 15 days of treatment, with final result HB II for the right and HB I for the left, in addition to vestibular compensation with progressive return to daily activities. After discharge, the brazilian therapeutic scheme was maintained, with no other medicines. She was referred for auditory rehabilitation with individual sound amplification device.

\section{Discussion}

Tuberculosis is a contagious bacterial disease, endemic in Brazil, which most commonly involves the lungs ( $75 \%$ to $90 \%$ cases) [1-3], with concomitant lesions present in $50 \%$ of the cases [4]. In head and neck, tuberculosis manifests predominantly in the larynx; less frequently in the middle and outer ear.

Tuberculous otitis media is an unusual, insidious and often under diagnosed secondary presentation [3], and may be considered a rare disease, with an estimated incidence of 0.05 to $2.7 \%$ of tuberculosis cases. The clinical picture is polymorphic, varying according to the patient's immunological status. Complications include deep sensorineural hearing loss, cranial nerve palsy, especially the facial nerve, abscesses, fistulas, and intracranial complications [4].

Tuberculous otitis media should be strongly suspected in all cases of chronic otorrhea that does not respond to antibiotics [5], 
especially in patients with current or previous tuberculosis disease [2]. Secondary bacterial infection caused by Staphylococcus, Pseudomonas, Klebsiella, Proteus and Streptococcus spp. interfere with the growth of Mycobacterium tuberculosis, hindering and delaying the diagnosis $[2,4,5]$.

The route of infection of the middle ear by tuberculosis is still discussed, being proposed, among others, hematogenous in miliary tuberculosis, by direct extension of the nasopharynx through the auditory tube, with or without primary infection in the pharynx, by external means through perforation of the tympanic membrane, by direct extension of adjacent structures, including intracranial focus via temporal bone [2-8].

Since the patient had multiple outbreaks of active infection due to tuberculosis, the route of dissemination considered in this case was hematogenic. There was no tuberculous meningitis or intracranial focus, confirmed by imaging and negative alcohol-acid resistant bacillus scan in the cerebrospinal fluid.

The classic characteristics of otologic tuberculosis were described by Wallmer in 1953: painless otorrhea, multiple perforations of the tympanic membrane, exuberant granulation tissue, hearing loss and bone necrosis [4], with other authors adding to the peripheral facial paralysis picture $72[3,4,7]$. Currently the disease presents mainly with significant otalgia, probably by the pressure of granulation tissue within the mastoid and serous or purulent otorrhea, being the most frequent finding $[2,7,8]$. It is common the formation of granulation tissue in the mastoid $[2,4,7,8]$. Otoscopy shows single or multiple perforations of the tympanic membrane, with exuberant pale or pink granulation tissue, which may be confused with cholesteatoma, in addition to polyps and purulent otorrhea $[2,4]$.

The patient in question presented otalgia, extensive tympanic membrane perforation and bilaterally abundant otorrhea, as well as bilateral facial paralysis and dizziness.

Hearing loss occurs in up to $90 \%$ of cases, usually severe and early [4]. It presents in a sensorineural, mixed or conductive way, which may persist after complete treatment of the infection [2,4]. In several situations hearing loss precedes inflammation of the tympanic membrane and the appearance of any other symptom [2].

In 2003 Werneck et al, described a case of miliary tuberculosis after initiation of the treatment for sudden deafness with corticoid in a 52-year-old female patient, suggesting, according to the authors, that there may be a relation between the presence of bacillus, although subclinically, and the emergence of sudden deafness by immunological mechanisms [9].

Rubio et al, in a review article in 2015 with patients diagnosed with aural or central nervous system tuberculosis with otoneurological manifestations, reported that $85.7 \%$ of patients had sensorineural hearing loss [4].

The patient had severe mixed right and left severe hearing loss, with little audio logical improvement after treatment. Considering that the patient presented bilateral suppurative labyrinthitis, the evolution was compatible with what is found in the literature, since suppurative labyrinthitis can lead to significant hearing loss [10], as presented.

Peripheral facial palsy is among the most common complications and is present in $40 \%$ of cases of tuberculous otitis media, being more frequent in children [4]. Its prognosis does not depend on decompression, but on early diagnosis and treatment. If the time between facial palsy and the initiation of treatment is less than five days, a full recovery should be expected. If treatment is started after two months of symptoms, there may be no recovery of the facial mimic $[2,4]$.

The Brazilian Ministry of Health advocates the treatment of new cases in adults of all forms of pulmonary and extra pulmonary tuberculosis (except meningogephalic form), infected or not with HIV with the basic regimen, composed of rifampicin, isoniazid, pyrazinamide and ethambutol. In fixed dose tablets of all four medicinal products [1].

The chemotherapy regimen against tuberculosis is the treatment of choice for a case of tuberculous otitis media [1,2], which improves the prognosis of most patients [8], and can prevent complications, especially if introduced early $[3,6]$.

\section{Conclusion}

We conclude that it is important to consider systemic diseases as a differential diagnosis in bilateral otologic disorders, especially those for which a high index of suspicion and specific investigation is necessary, including other sites of the disease, such as tuberculosis.

\section{References}

1. Ministerio Da Saude. Manual de Recomendações para o Controle da Tuberculose no Brasil. Brasília (DF). 2011.

2. Pinho MM, Kos AO. Otite média tuberculosa. Rev. Bras. Otorrinolaringol. 2003; 69: 829-837.

3. Gupta N, Dass A, Goel N, Tiwari S. Tuberculous Otitis Media Leading to Sequentialib Bilateral Facial Nerve Paralysis. Iran J Otorhinolaryngol. 2015; 27: 231-237.

4. Diplan R, Johanna M, et al. Neuro-otologic manifestations of tuberculosis. "The great imitator". Am J Otolaryngol. 2015; 36: 467-471.

5. Rajesh Gandham N, Sardar M, Jadhav SV, Vyawahare C, Misra R. Tuberculous Otitis with Proteus Mirabilis Colnfection: An Unsuspected Presentation Encountered in Clinical Practice. J Clin Diagn Res. 2014; 8: 1-3.

6. Prakash M, Carlton Johnny J. Intra Cranial Complications of Tubercualous Otitis Media. J Pharm Bioallied Sci. 2015; 7: 51-54.

7. Sens PM, Almeida CIR, Valle LO, Costa LHC, Angeli MLS. Tuberculose de orelha, doença profissional? Rev.Bras. Otorrinolaringol. 2008; 74: 621-627.

8. Hwang GH, Jung JY, Yum G, Choi J. Tuberculous Otitis Media with Facial Paralysis Combined with Labyrinthitis. Korean J Audiol. 2013; 17: 27-29.

9. Werneck ALS, Gurgel LCA, Mello LM, Albuquerque GQ. Sudden sensorineural hearing loss: A case report supporting the immunologic theory. Arq. Neuro-Psiquiatr. 2003; 61: 1018-1022.

10. Kaya S, Tsuprun V, Hızı Ö, Paparella MM, Cureoglu S. Quantitative Assessment of Cochlear Histopathologic Findings in Patients With Suppurative Labyrinthitis. JAMA Otolaryngol Head Neck Surg. 2016; 142: 364-369. 